\title{
Genetic Reassortment of Rice stripe virus RNA Segments Detected by RT-PCR Restriction Enzyme Analysis-based Method
}

\author{
Miranda Gilda Jonson ${ }^{1,2}$, Sen Lian ${ }^{2}$, Hong-Soo Choi ${ }^{3}$, Gwan-Seok Lee ${ }^{3}$, Chang-Suk Kim ${ }^{3}$ and Kook-Hyung Kim ${ }^{2 *}$ \\ ${ }^{I}$ Department of Biology, School of Science and Engineering, Ateneo de Manila University, Katipunan Avenue, Loyola Heights \\ Quezon City 1108, Philippines \\ ${ }^{2}$ Department of Agricultural Biotechnology and Plant Genomics and Breeding Institute, College of Agriculture and Life Sciences, \\ Seoul National University, Seoul 151-921, Korea \\ ${ }^{3}$ Department of Agricultural Biology, National Academy of Agricultural Science, Rural Development Administration, Suwon 441- \\ 707, Korea
}

(Received on March 22, 2011; Accepted on April 11, 2011)

Our previous sequence and phylogenetic analyses of the Korean Rice stripe virus (RSV) suggested possible genetic reassortment of RNA segments, but whether this RNA variation contributed to the recent RSV outbreaks in Korea is yet unclear. To further clarify these RSVRNA segment variations, we developed a reverse transcription-polymerase reaction/restriction enzyme (RTPCR/RE) analysis-based method. We identified five REs, including DraI, EcoR1, NdeI/AseI, and SpeI, that could differentiate RSV RNA 1-4 subtypes, respectively. Our RT-PCR/RE results provided a clear pattern of RNA reassortment, i.e., different groups of isolates having their RNA segments derived from two to three different RSV ancestors, such as from Eastern and Southwestern Chinese or Japanese $M$ and $T$ isolates. We also found that the migratory small brown planthopper from Eastern China caught by aerial net traps that possesses RSVRNA3 genotypes corresponds mainly to Eastern China, with a few for Southwestern China based on RT-PCR/ $\mathrm{RE}$, sequence and phylogenetic analyses, indicating that RSV populations in Eastern China may also have strong RNA variation. The development of an $R E$ analysisbased method proved a useful epidemiological tool for rapid genotyping and identification of mixed infections by RSV strain and by different subtype.

Keywords : genotyping, genetic structure, phylogenetic relationships, restriction enzyme analysis, Rice stripe virus

The genome of Rice stripe virus (RSV), the prototype member of the genus Tenuivirus, consists of four ssRNAs, with a total genome size of about $23 \mathrm{~kb}$ (Fig. 1; Toriyama and Tomaru, 1995). RNA1 is the largest segment, having negative sense, while the other three segments are ambisense,

\footnotetext{
*Corresponding author.

Phone) +82-2-880-4677, FAX) +82-2-873-2317

E-mail)kookkim@snu.ac.kr
}

whereby a single protein is encoded at the 5 proximal end in viral (v) and viral complementary (vc) RNA strands (Hamamatsu et al., 1993; Takahashi et al., 1993; Zhu et al., 1991, 1992). The RSV genome has a total of seven proteins and most of their functions are known, except for NS2 coded in vRNA2. The function of proteins coded in vcRNA1, vcRNA2, vcRNA3, and vRNA4 have long been known as RNA-dependent RNA polymerase (RdRp; Toriyama et al., 1994), glycoprotein-membrane protein (Takahashi et al., 1993), nucleocapsid protein, and noncapsid protein or disease-specific protein (Kakutani et al., 1990; Zhu et al., 1992), respectively. However, proteins coded in vRNA3 and vcRN4 were only recently reported as suppressors of posttranscriptional gene silencing (PTGS; Hemmes et al., 2007) and movement protein (Xiong et al., 2008), respectively.

RSV-infected plants usually show chlorotic stripes on the leaves and stunted growth, while severely infected seedlings are lethal. The virion morphology of the causal virus has circular filament and is actively and persistently transmitted by the small brown planthopper (SBPH) Laodelphax striatellus (Koganezawa, 1977). RSV outbreaks have also occurred in China, Japan, Taiwan, and the former USSR (Hibino, 1996).

For insect transmitted viruses like RSV, its spread is mainly dependent on vector migration. In a previous study on the Nilaparvata lugens (Stal), migration processes in Korea using the boundary layer atmospheric model and a geographic information system showed that the main source of insects immigrated into South Korea in the early part of June were from the Eastern part of China (Zhu et al., 2000). China and Japan are in close proximity to Korea, and reports show that China has a longer history of RSV outbreaks than Japan (Wei et al., 2009).

During 2007-2009, RSV had burgeoning incidences that caused significant yield losses, posing an enigma to rice growers and many rice scientists in South Korea. The cause 
of the sudden reemergence of RSV is undetermined, although several possible reasons exist causing outbreaks, such as change in host genetic susceptibility (Garcia-Arenal et al., 2003; Gu et al., 2007; Jeger et al., 2006; Miranda et al., 2000) variations of virulence among RSV isolates due to mutations and RNA reassortment of RNA segments, and change in insect population biotypes (Diehl and Bush, 1984; Myint et al., 2009). Although results of a previous phylogenetic study showed clustering of Korean RSV isolates into different subtypes, the lineages of RSV isolates and those of Chinese and Japanese isolates vary from RNA to RNA segment, suggesting the occurrence of possible genetic reassortment (Jonson et al., 2009a, 2009b). Direct evidence, however, is still lacking and the origin of RSV isolates in Korea is also unclear.

The use of sequence analysis to differentiate virus isolates is laborious, time-consuming, and costly. In this present study, we have developed a rapid, simple genotyping method that could be used to differentiate the RSV-RNA genome subtypes and to facilitate easy monitoring of the RSV strains currently circulating in the field. Data collected using this technique have confirmed the origin of migratory SBPHs caught in Jeonbuk and Chungnam provinces and showed evidence of the mechanism of genetic change of the RSV population structure in Korea.

\section{Materials and Methods}

Plants, insect virus samples, and total RNA extraction. About 100 randomly selected RSV isolates collected in four major provinces of Korea [Jeonbuk (JB), Jeonnam (JN), Chungnam (CN), and Jeju (JJ)] during 2007-2009 and from individual migratory SBPHs (13 insect samples in total) caught by aerial net traps in early June in JB and CN provinces thought to have migrated from Eastern China were analyzed in this study. Total RNA from infected plants and insects was extracted following the method of a previously reported study (Jonson et al., 2009a, 2009b).

RT-PCR, identification, and analysis of unique restriction enzyme sites. We identified the possible location of unique restriction sites by using the primers and RT-PCR protocols in our previous studies (Jonson et al., 2009a, 2009b). Due to the relatively large sizes of RNAs 1 and 2 segments, however, we have prioritized specific regions with relatively high nucleotide (nt) sequence variations. These regions include the terminal region of RNA1 at nt 7413-8970 and initial region of RNA2 at nt 1-1333. For RNA3 and RNA4 segments, full-length segments were amplified. Fig. 1 shows the specific regions of RNAs 1 and 2 and the full-length of RNA3 and RNA4 segments. The primer sets used were as follows: RNA1-F8 (nt 7413-
8970) primer sets: RS1-15F, 5'-CCATGTAGACCCTAAAGCRAACT-3' and RS1-16R 5'-ACACAAAGTCCAGAGGAAAACAA-3' with an expected fragment size of $1.5 \mathrm{~kb}$; RNA2-F1 (nt 1-1333) primer sets: RS2-17F 5'-ACACAAAGTCCTGGGTATATAAG-3' and RS2-18R 5'-GAGGGATTGGCAGTTTGCCCTAT-3' with 1.3-kb product; RNA3 primer sets: RS3-23F 5'-ACACAAAGTCCTGGGTAAAATAG-3' and RS3-26R 5'-ACACAAAGTCWGGGTAATAAAAT-3' with a 2.5-kb product; and RNA4 primer sets: RS4-27F 5'-ACACAAAGTCCAGGGCA-3' and RS4-30R 5'-ACACAMAGTCAKGGYAT-3' with a $2.1-\mathrm{kb}$ product. The DNA fragments were then analyzed using NEBcutter v. 2.0 (http://tools.neb.com/NEBcutter2/index.php) to find a unique RE site that could differentiate RSV subtypes.

RNA3 cloning, sequencing, and phylogenetic analyses. The RNA3 sequences from the new collected leaf and insect infected samples representing each RNA subtype were initially analyzed using the BioEdit sequence alignment editor, version 7.0.9 (Hall, 1999), and the phylogenetic and molecular evolutionary analyses were conducted using MEGA version 4 (Tamura et al., 2007). Phylogenetic relationships based on RNA3 nucleotide sequences of eight

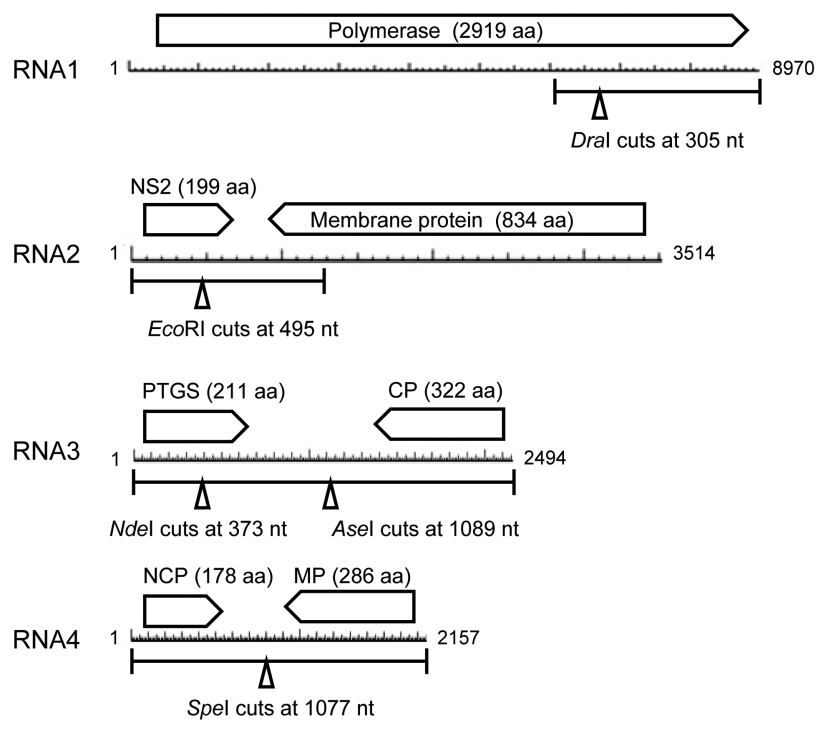

Fig. 1. Schematic representation of the genome organization of Rice stripe virus (RSV). The graduated lines represent the RNA segments, the rectangular arrowed boxes represent the gene encoded, and the values enclosed are the number of amino acid at viral and viral complementary-sense RNAs. Bar-ended lines in RNAs 1-4 show the specific region/genome amplified, and triangles point to the location of differentiating restrictions enzymes used to differentiate RSV subtypes. RNA1 fragment (nt 7413-8970) contained a part of the 5'-terminal region of the RdRp gene and the complete 3'-terminal noncoding region. RNA2 fragment (nt 1-1333) contained the 5' noncoding region, NS2 protein, and an intergenic region; the 3'-terminal region of Nvc2 codes for a membrane protein. 
Korean (six plants and two insect RSV-infected samples), Chinese (five isolates), and Japanese (two isolates) isolates were established using the neighbor-joining method with a bootstrap value for each internal node based on 1,000 random replications. The Chinese and Japanese-RSV isolates' accession numbers that we have included in our analysis were as follows: D01094, the Japanese-M (Kakutani et al., 1990, 1991); NC_003776, Japanese-T (Toriyama, 1994); EU931524, EU931520, and EU931516 for ABBO7, HKF07, and JYCO7 (Genbank, unpublished), respectively; EF141329, JY (Genbank, unpublished); DQ333944, Z (Zhang et al., 2007); and Y11095.1, Y (Qu et al., 1997). All Chinese isolates are from Eastern China and only isolate Y was from Southern China.

\section{Results}

Subtype-specific restriction patterns. To test the efficiency of our newly developed RE analysis, we reanalyzed the 13 Korean RSV isolates from our previous phylogenetic studies of RNAs 1 and 2 (Jonson et al., 2009a) and RNAs 3 and 4 (Jonson et al., 2009b).

The RNA1 fragment contained a part of the $\mathrm{C}$ terminal region of the $\mathrm{RdRp}$ gene and the full 3 ' terminal region. The differentiating RE identified was DraI, which cuts at nt 305 of the $\mathrm{C}$ terminal region of the RdRp protein (Fig. 1). In our previous report (Jonson et al., 2009a), RNA1 phylogenetic tree showed two major clades: one clade consisted of nine isolates originated from Korea and grouped together with Eastern China RSV isolates which we referred this RSV population as IA. This IA population do not have the DraI site as shown in our Fig. 2A. The second clade consisted of the rest of the Korean RSV isolates and together with those of Japanese $\mathrm{T}$ and Eastern China which we referred this RSV population as IIC. This IIC population as shown in our RE analysis has the DraI site (Fig. 2A). However, isolate SA-JN showed a different digestion product size, indicating that the DraI in this isolate was located at a different position in the fragment.

The RNA2 fragments contained the 5' noncoding region, NS2 protein, and an intergenic region; the C-terminal region of Nvc2 codes for a membrane protein (Fig. 1). In our previous study (Jonson et al., 2009a), RNA2 was reported to have three subtypes, but in this study based on RE analysis, it appeared to have only two differentiating subtypes. The Korean isolates under subtypes I and II in previous analysis possessed the EcoRI sites and these isolates have IIC origin. On the other, the Korean isolates under subtype III in previous study did not have any EcoRI site and these isolates have IA origin. The two subtypes of RNA2 having IA and IIC origin were differentiated using EcoRI, which was located at nt 495 on the NS2 gene.
Although, based on phylogenetic analysis, isolate IS-JB belongs to the group without an RE site. Unexpectedly, however, according to the RE analysis, it contained EcoRI but with different sizes of digested products (Fig. 2B).

For RNA3, we identified two differentiating REs, NdeI and AseI, representing each of the ancestral isolates. NdeI cuts at nt 373 , which is located within the central region of the NS3 gene that codes for a suppressor of PTGS, while Ase I cuts at nt 1089 and is located in the intergenic region (Fig. 1). Results of the RNA3-RE analysis of Korean

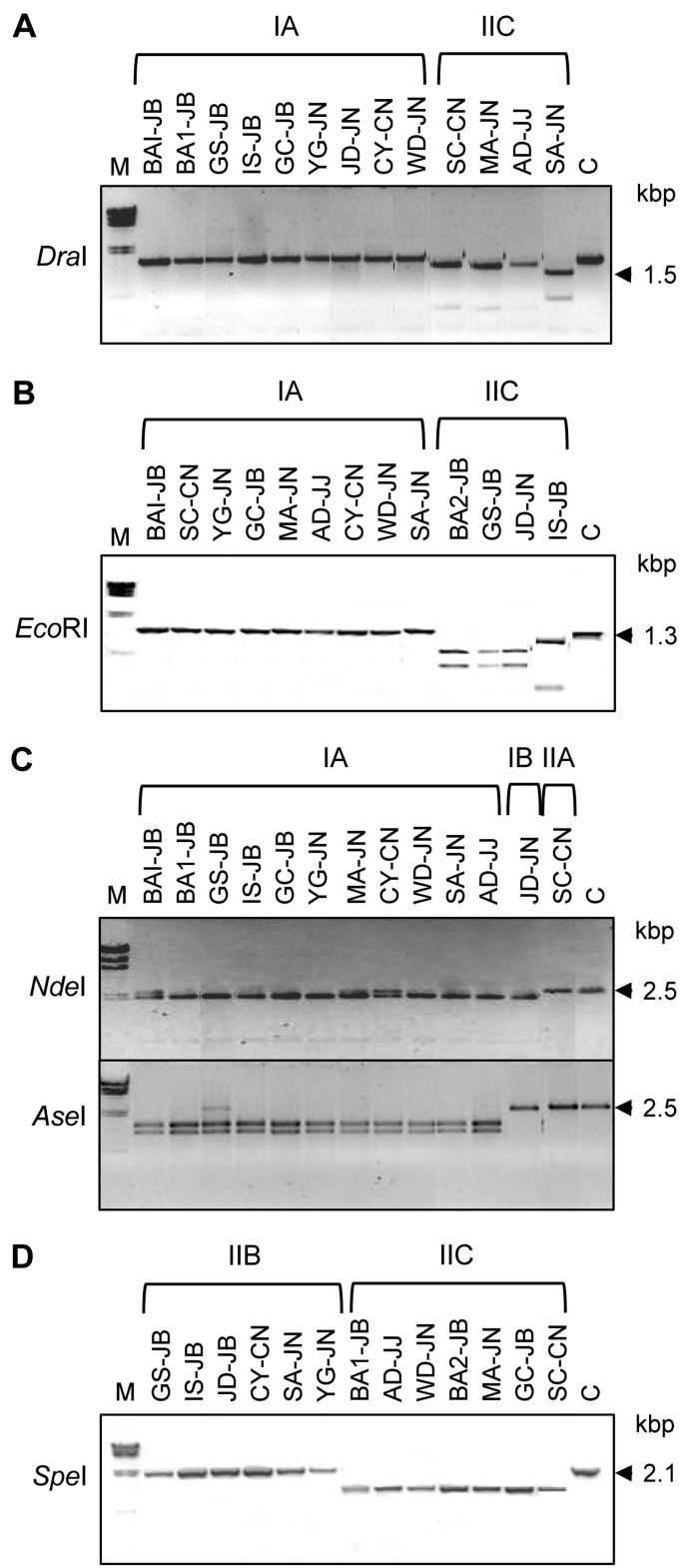

Fig. 2. Restriction enzymes analyses of different Korean RSV isolates RNA1 (A), RNA2 (B), RNA3 (C), and RNA4 (D) digested with DraI, EcoRI, AseI/NdeI, and SpeI, respectively. M is the HindIII-digested lambda DNA size marker and C, control or undigested amplified DNA product. 
isolates were congruent with the previous phylogenetic analysis (Jonson et al., 2009b); however, unexpectedly, we found one isolate (JD-JN) that grouped with those of Eastern China by phylogeny, but in RE analysis showed similar results to the Southwestern China isolate, which we referred this RSV population as IB having a sequence with NdeI but without AseI (Fig. 2C).

The differentiating RE identified for Korean RSV-RNA4 was SpeI, which revealed two distinct subtypes such as derived from IIC and isolates derived from Japan-M isolate referred as IIB. This result had high congruence with the previous phylogenetic analysis (Jonson et al., 2009b). In the same report, the phylogenetic analysis of Korean RSVRNA4 showed four major clusters. However, based on RE analysis in this study, the two previously identified subtypes I and II, belong under single group (Fig 2D).

In all cases, all REs used in this study showed distinct restriction patterns after $3 \mathrm{~h}$ digestion and $30 \mathrm{~min}$ gel $(1.0 \%)$ migration to distinguish each of the five subtypes (Fig. 2A, 2B, 2C-AseI). However, except for NdeI, they required longer (overnight digestion) incubation to obtain complete digestion of RNA3 PCR-amplified dsDNA, and the restriction products necessitated a longer period of electrophoresis $(40 \mathrm{~min}$ ) for clear separation of the subtypes (Fig. 2C-NdeI and Fig. 3-NdeI). The relationships between restriction maps and different RSV subtypes are shown in Table 1.

Origin of RSV in Korea. The origin of RSV in Korea is not fully understood. To clarify this point, we tested individual migratory SBPHs caught by aerial net traps in early June in JB and CN provinces. These insects were thought to have migrated from Eastern China, based on the sudden, massive interception in 2009 (unpublished data). Fourteen SBPHs from Buan JB (BA-JB) and Taean CN (TA-CN) provinces were tested with RT-PCR to detect the RNA3 segment. A full-length RNA3 segment, with a size of $2.5 \mathrm{~kb}$, was amplified from 13 of 14 SBPHs using RTPCR. The RNA3 cDNA of the 13 insects was amplified and treated with AseI and NdeI. Results showed that the digestion pattern of the 12 samples resembled that of Eastern China, only one sample from BA-JB showed a digestion pattern similar to that of Southwestern China, and none of the samples showed a digestion pattern similar to that of Japan (Fig. 3).

Due to quarantine issues, we are not able to conduct $\mathrm{RE}$ digestions for all isolates from outside Korea; instead their sequences available in GenBank were analyzed using NEBcutter v. 2.0 (free online software). Based on sequences analyzed using NEBcutter software, we found that the China JY and Z isolates had AseI and NdeI sites and these isolates were obtained from Eastern China. While the China Y isolate, which was obtained from Southwestern China, had NdeI but no AseI. However, Japanese M and T isolates do not have these two RE sites.

Genetic reassortment. Table 2 summarizes the genetic structure of RSV in Korea based on RE analysis of the RNA 1-4 segments. Results further revealed that the genetic structure of the RSV population in Korea consisted of two major RSV types, namely Type I coming from China and Type II from Japan. Type I includes two subtypes, IA and IB, which originate from Eastern and Southwestern China, respectively. However, Type II consisted of three subtypes,

Table 1. Correlation between restriction maps and the 13 Korean-Rice stripe virus isolates subtypes

\begin{tabular}{|c|c|c|c|c|c|}
\hline Genome & $\mathrm{RE}$ & Isolates & $\begin{array}{l}\mathrm{RE} \\
\text { site }\end{array}$ & Restriction map (kbp) & $\begin{array}{l}\text { Sub } \\
\text { type }\end{array}$ \\
\hline RNA1 & DraI & $\begin{array}{c}\text { GS, JD, BA2,CY, WD, BA1, IS, GC, YG } \\
\text { SC, AD, MA /SA }\end{array}$ & $\begin{array}{l}- \\
+\end{array}$ & $\begin{array}{c}1.5^{\mathrm{a}} \\
0.3,1.19 / 0.4,1.1^{\mathrm{b}}\end{array}$ & $\begin{array}{l}\text { IA } \\
\text { IIC }\end{array}$ \\
\hline RNA2 & EcoRI & $\begin{array}{c}\mathrm{SA}, \mathrm{JD}, \mathrm{SC}, \mathrm{AD}, \mathrm{MA}, \mathrm{CY}, \mathrm{WD}, \mathrm{BA} 1, \mathrm{IS}, \mathrm{GC}, \mathrm{YG} \\
\text { GS, BA2, JC / IS }\end{array}$ & + & $\begin{array}{c}1.3^{\mathrm{c}} \\
0.5,0.8 / 0.3,1.0^{\mathrm{d}}\end{array}$ & $\begin{array}{c}\text { IA } \\
\text { IIC/IA }\end{array}$ \\
\hline RNA3 & Nde1/Ase1 & $\begin{array}{c}\mathrm{SC} \\
\mathrm{JD}\end{array}$ & $\begin{array}{l}-/- \\
+/+ \\
+/-\end{array}$ & $\begin{array}{l}2.5 / 2.5^{\mathrm{e}} \\
0.3,2.2 / 1.4,1.1^{\mathrm{f}} \\
0.3,2.2 \mathrm{f} / 2.5^{\mathrm{f}}\end{array}$ & $\begin{array}{l}\text { IIA } \\
\text { IA } \\
\text { IB }\end{array}$ \\
\hline RNA4 & SpeI & $\begin{array}{c}\text { GS, SA, JD, CY, IS, YG } \\
\text { SC, BA2, AD, MA, WD, BA1, GC }\end{array}$ & $\begin{array}{l}- \\
+\end{array}$ & $\begin{array}{c}2.1^{\mathrm{g}} \\
1.1,1.0^{\mathrm{h}}\end{array}$ & $\begin{array}{l}\text { IIB } \\
\text { IIC }\end{array}$ \\
\hline
\end{tabular}

\footnotetext{
a,b Uncut and cut RT-PCR product after digestion with DraI, respectively.

$c, \mathrm{~d}$ Uncut and cut RT-PCR product after digestion with $E c o$ RI, respectively.

e,f Uncut and cut RT-PCR product after digestion with NdeI/AseI, respectively.

${ }^{g, h}$ Uncut and cut RT-PCR product after digestion with SpeI, respectively.
} 


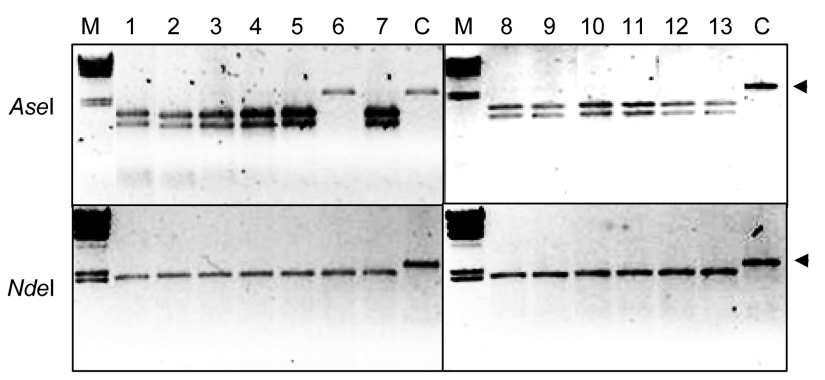

Fig. 3. Restriction enzyme analysis of amplified RNA3 from viruliferous insects. Total RNA was extracted from RSV-infected individual small brown planthoppers caught by aerial net traps in Buan, Jeonbuk (insect nos. 1-7) and Taean, Chungnam (insect nos. 8-13) provinces in early June 2009 and their amplified PCR product digested with AseI and NdeI. M and C represent the HindIII-digested lambda DNA size marker and the 2.5 -kbp undigested PCR amplified RNA3 genomic DNA (arrow), respectively.

IIA, IIB, and IIC, that originated from a mixture of Japanese $\mathrm{M}$ and $\mathrm{T}$ isolates, Japanese $\mathrm{M}$ isolate alone, and mixtures of Japanese $\mathrm{T}$ and Eastern China isolates. These mixtures of RNAs coming from different ancestors are an indication of the occurrence of natural genetic reassortment among Korean RSV isolates. The pattern of RNA reassortment is clearly shown in Table 3. Isolates consisting of two different RSV ancestors were CY-CN, WD-JN, BA1-JB, IS-JB, and GCJB; their RNAs 1-3 have a common ancestor (IA, Eastern China) but the RNA4 originated from different ancestors, either from Japanese M (IIB) or mixtures from Japan and China (IIB and IIC). Another group of isolates having RNA reassortment coming from two different ancestors consisted of BA2-JB, AD-JJ, and MA-JN. High RNA reassortment coming from three different ancestors was found in isolates GS-JB, SA-JN, JD, JN, and SC-CN.

RNA3 sequence and phylogenetic analysis. Since RNA3 phylogenetic analyses showed distinct ancestral derivation, we applied RNA3-RE analysis to further confirm the identity of the new samples representing each RNA subtype. We amplified the RNA3 full-length region of about 100 new RSV-infected plants collected in four major provinces of Korea (JB, JN, CN, and JJ) and 14 viruliferous samples collected from JB and $\mathrm{CN}$ provinces, all obtained from 2007 to 2009, and each amplicon was genotyped using the RE analysis method. Results of RE analyses further demonstrated that the genetic structure of RSV in Korea is composed of mixtures of RSV subtypes coming from different ancestors (infected plants, data not shown; viruliferous insects, Fig. 3). Based on RE analyses, we selected a total of three isolates representing subtype IA obtained from infected plant samples (BA-JB16, JD-JN6) and a viruliferous insect (TA-CN5); four isolates representing subtype IB, i.e., three samples obtained from infected plants (SC-CN6,
Table 2. Genetic structure of Korean isolates of RSV showing their possible origin and ancestral isolates

\begin{tabular}{ccl}
\hline \hline Typea & Subtype & \multicolumn{1}{c}{ Origin } \\
\hline I & A & Eastern China RSV isolates \\
& B & Southwestern China RSV isolates \\
II & A & Japan M and T isolates \\
& B & Japan-M isolate \\
& C & Japan-T and China Eastern isolates \\
\hline
\end{tabular}

${ }^{a}$ Type I consists of China-RSV ancestors while type II includes Japan (M and $\mathrm{T}$ ) and Eastern China ancestors.

SC-CN70, and JD-JN4) and an insect sample (BA-JB6); and a single sample for subtype II obtained from infected plants (GS-JB16). We determined the RNA3 complete sequence of these selected samples, and were submitted to Genbank under accession numbers HQ343287-HQ343294. Results of our phylogenetic analysis showed that these selected isolates grouped according to their subtype (Fig. 4), which further confirmed that the RE analysis developed in this study is an efficient and reliable genotyping method.

\section{Discussion}

Plant viruses with an RNA genome are prone to mutation (Garcia-Arenal et al., 2003; Jeger et al., 2006). On the other hand, segmented plant ( $\mathrm{Gu}$ et al., 2007; Miranda et al., 2000) and animal RNA viruses (Henderson et al., 1995; Rodrigues et al., 1998) commonly undergo genetic reassort-

Table 3. Evidence for RNA Reassortment within Korean isolates of RSV based on analyses of sequence, phylogenetic and RE showing its distinctive ancestors ${ }^{\mathrm{a}}$

\begin{tabular}{lcccc}
\hline \hline Isolates & RNA1 & RNA2 & RNA3 & RNA4 \\
\hline GS-JB & IA $^{\text {b }}$ & IIC & IA & IIB \\
SA-JN & IIC & IA & IA & IIB \\
JD-JN & IA & IA & IB & IIB \\
SC-CN & IIC & IA & IIA & IIC \\
BA2-JB & IA & IIC & IA & IIC \\
AD-JJ & IIC & IA & IA & IIC \\
MA-JN & IIC & IA & IA & IIC \\
CY-CN & IA & IA & IA & IIB \\
WD-JN & IA & IA & IA & IIC \\
BA1-JB & IA & IA & IA & IIC \\
IS-JB & IA & IA & IA & IIB \\
GC-JB & IA & IA & IA & IIC \\
YG-CN & IA & IA & IA & IIB \\
\hline
\end{tabular}

${ }^{a}$ Isolates collected in 2007-2009.

${ }^{\mathrm{b}}$ Each type represents as follows: IA, Eastern China; IB, South Western China; IIA, Japan-M and -T; IIB, Japan-M; IIC, Japan T and 1A; Subtype IA showed the predominant subtype found in Korea. 


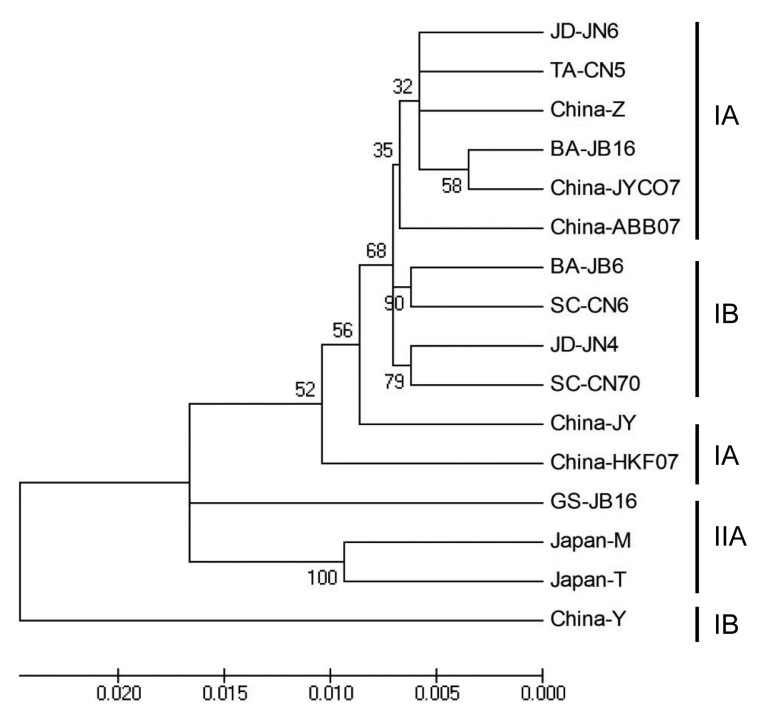

Fig. 4. Phylogenetic tree based on RNA3 nucleotide sequences of the new Korean RSV samples collected during 2007-2009, including Chinese and Japanese isolates. The clustering obtained from phylogenetic analysis corresponds to the subtypes identified by RE analysis. The tree was constructed with the neighborjoining algorithm and a bootstrap value for each internal node based on 1,000 random replications using the Kimura twoparameter model implemented in MEGA version 4 (Tamura et al., 2007). Of eight Korean isolates, only two samples were obtained from insects (TA-CN5 and BA-JB6); the rest were all obtained from RSV-infected rice plant samples. Included in the analysis were 5 Eastern China- (Z, JY, JYCO7, ABBO7, and HKFO7), 1 Southwestern China (Y) and 2 from Japan (M and T) RSV isolates.

ment wherein RNA segments reassort, thereby generating new variants. For this reason, reassortment of an RNA virus with a divided genome has been proposed to be an important mechanism in the evolution of plant RNA viruses (GarciaArenal et al., 2003; Jeger et al., 2006; Roossinck, 1997). Previous results from our laboratory (Jonson et al., 2009a, 2009b) have suggested some clues of possible genetic reassortment causing the sudden reemergence of RSV, which became a major rice virus disease in Korea during 2007-2009. Here, we further clarified RNA variations by developing RT-PCR/RE, a rapid genotyping method capable of distinguishing the different RNA subtypes. We also clarified the origin of RSV in Korea by testing viruliferous insects of known origin using RT-PCR/RE, sequencing, and phylogenetic analyses.

The relationships of Korean RSV-RNAs 1, 2, and 4 (Figs. 2A, B, and D) to the Chinese and Japanese isolates were not distinct; hence, they were variably grouped depending on subtype, suggesting that Korean RSV-RNA 1, 2, and 4 isolates may be derived from mixtures of two or more ancestors. In this study, the natural genetic reassortment among Korean RSV isolates was clearly presented and their distinct ancestors identified (Table 2). The overall phylogenetic and
RT-PCR-RE analyses of RNA 1-4 sequences of different RSV isolates obtained from various geographical areas in Korea revealed two major RSV types, referred to as of Chinese (I) and Japanese (II) origin. These major types showed a total of five different subtypes. Type I consisted of two subtypes referred as Eastern Chinese (IA) and Southwestern Chinese (IB), while Type II consisted of three subtypes, referred as a mixture of Japanese M and T (IIA), Japanese M (IIB), and a mixture of Japanese $T$ and Eastern Chinese (IIC).

The RE analysis of our previously analyzed complete genome (Jonson et al., 2009a, 2009b) of 13 Korean RSV isolates showed that the predominant subtype of each RNA genome was composed of Eastern Chinese descendents (IA, Table 3). Similarly, in this study, the newly analyzed RNA3 segment of RSV isolates collected during 20072009 were predominantly Eastern Chinese type (data not shown). However, in a study by Wei et al. (2009), the RSV population in Eastern China was composed of only two subtypes, while the Southwestern China types were shown to consist mainly of a single subtype and a small proportion of subtypes found in Eastern China, indicating that the Southwestern China RSV population is more diverse than that of Eastern China. Our genetic profile results, however, based on sequence and RE analyses obtained from SBPHs caught in JB and CN provinces in Korea that were thought to have migrated from Eastern China showed electrophoretic patterns predominantly similar to those of Eastern China, except for a single insect sample that showed characteristics of Southwestern Chinese isolates. This result suggests that the RSV population in Eastern China may also be experiencing high RNA variation. In addition, the 100 new randomly selected RSV-infected plants collected in four major provinces of Korea and collected during 2007-2009 that were analyzed in this study also showed that the Eastern Chinese genotypes were the dominant subtypes. These results may provide an insight showing that, most likely, the initial genetic structural origin of the RSV population in Korea is composed mainly of Eastern Chinese isolates.

Note that the consecutive RSV outbreaks in China started in 2002 (Wei et al., 2009), while those in South Korea started in 2007 (Jonson et al., 2009a, 2009b). Consequently, our data further suggest that the sudden reemergence of RSV in Korea was most likely triggered by the invasion of new subtypes due to migratory insects from Eastern China. These new subtypes are composed of mixtures of subtypes, that is an isolate derived from different ancestors (Eastern and Southwestern), and subsequently may have caused genetic changes through exchanges of RNA segments from one isolate to another coming from different RSV sources or ancestors, as demonstrated in this study (Table 3). 
Although Japanese RSV isolates were also found in Korea, we do not have direct evidence of insects migrating from Japan to Korea. Pender (1994) mentioned several studies on the potential movement of rice planthoppers among temperate countries that is, from China to Korea and Japan. Hence, the possibility of insects that migrated from China might carry the genotype of Japanese isolates could not be ruled out in this study. The distribution of RSV subtypes between Korean and Chinese populations, however, is contradictory. RSV subtypes in China showed a correlation with their geographical locations (Wei et al., 2009), while the distribution of Korean RSV subtypes was not correlated with their geographical location, as also found for Cucumber mosaic virus (CMV), another segmented RNA plant virus (Fraile, 1997).

Previous studies have utilized a variety of molecular methods to detect naturally occurring and laboratory-generated reassortants (Gu et al., 2007; Uyeda et al., 1995). The RT-PCR/RE analysis-based method has been effectively used in detecting naturally occurring genetic reassortment (Chen et al., 2007; Klimov and Cox, 1995). In this study, the results of our phylogenetic analysis of selected subtypes obtained from RE analysis showed clustering of isolates having similar subtypes to those of Chinese and Japanese ancestors, further confirming the efficiency of the RE method in differentiating subtypes (Fig. 4). RE analysis was utilized to rapidly differentiate RSV-RNA genome subtypes and will be used to screen field RSV-infected plant and insect samples to facilitate easy monitoring of RSV strains currently circulating in the field. The development of a RE analysis-based method proved to be a useful epidemiological tool for rapid identification of mixed infections by RSV strains and by different subtypes.

\section{Acknowledgements}

This research was supported in part by a grant from the Rural Development Administration (No. PJ007009); the Korea Institute of Planning and Evaluation for Technology of Food, Agriculture, Forestry and Fisheries (No. 10810302); and the Vegetable Breeding Research Center (No. 710001-03) through Agriculture Research Center program from the Ministry for Food, Agriculture, Forestry and Fisheries, Republic of Korea. MGJ was supported by a post-doctoral research fellowship from the MEST through the Brain Korea 21 Project.

\section{References}

Chen, Y., Chen, J., Zhang, H., Tang, X. and Du, Z. 2007. Molecular evidence and sequence analysis of a natural reassortant between Cucumber mosaic virus subgroup IA and II strains.
Virus Genes 35:405-413.

Diehl, S. R. and Bush, G. L. 1984. An evolutionary and applied perspective of insect biotypes. Annu. Rev. Entomol. 29:471504.

Fraile, A., Alonso-Prados, J. L., Aranda, M. A., Bernal, J. J., Malpica, J. M. and Garcia-Arenal, F. 1997. Genetic exchange by recombination or reassortment is infrequent in natural populations of a tripartite RNA plant virus. J. Virol. 71:934-940.

Garcia-Arenal, F., Fraile, A. and Malpica, J. M. 2003. Variation and evolution of plant virus populations. Int. Microbiol. 6:225-232.

Gu, H., Zhang, C. and Ghabrial, S. A. 2007. Novel naturally occurring Bean pod mottle virus reassortants with mixed heterologous RNAlgenomes. Phytopathology 97:79-86.

Hall, T. A. 1999. BioEdit: a user-friendly biological sequence alignment editor and analysis program for Windows 95/98/ NT. Nucl. Acids Symp. Ser. 41:95-98.

Hamamatsu, C., Toriyama, S., Toyoda, T. and Ishihama, A. 1993. Ambisense coding strategy of the rice stripe virus genome: in vitro translation studies. J. Gen. Virol. 74:1125-1131.

Hemmes, H., Lakatos, L. Goldbach, R., Burgyan, J. and Prins, M. 2007. The NS3 protein of Rice hoja blanca tenuivirus suppresses RNA silencing in plant and insect hosts by efficiently binding both siRNAs and miRNAs. RNA 13:1079-1089.

Henderson, W. W., Monroe, M. C., St. Jeor, S. C., Thayer, W. P., Rowe, J. E., Peters, C. J. and Nichol, S. T. 1995. Naturally occurring $\operatorname{Sin}$ nombre virus genetic reassortants. Virology 214:602-610.

Hibino, H. 1996. Biology and epidemiology of rice viruses. Annu. Rev. Phytopathol. 34:249-274.

Jeger, M. J., Seal, S. E. and van den Bosch, F. 2006. Evolutionary epidemiology of plant virus disease. Adv. Virus Res. 67:163203.

Jonson, G.-M., Choi, H.-S., Kim, J.-S., Choi, I.-R. and Kim, K.-H. 2009a. Sequence and phylogenetic analyses of the RNA1 and RNA2 segments of Korean Rice stripe virus isolates and comparison with those of China and Japan. Arch. Virol. 154:17051708.

Jonson, G.-M., Choi, H.-S., Kim, J.-S., Choi, I.-R. and Kim, K.-H. 2009b. Complete genome sequence of the RNAs 3 and 4 Segments of Rice stripe virus isolates in Korea and their phylogenetic relationships with Japan and China isolates. Plant Pathol. J. 25:142-150.

Kakutani, T., Hayano, Y., Hayashi, T. and Minobe, Y. 1990. Ambisense segment 4 of Rice stripe virus: Possible evolutionary relationship with phleboviruses and uukuviruses (Bunyaviridae). J. Gen. Virol. 71:1427-1432.

Kakutani, T., Hayano, Y., Hayashi, T. and Minobe, Y. 1991. Ambisense segment 3 of Rice stripe virus: The first instance of a virus containing two ambisense segments. J. Gen. Virol. 72:465-468.

Klimov, A. I. and Cox, N. J. 1995. PCR restriction analysis of genome composition and stability of cold-adapted reassortant live influenza vaccines. J. Virol. Methods 52:41-49.

Koganezawa, H. 1977. Purification and properties of rice stripe virus. Trop. Agric. Res. Ser. 10:151-154. 
Miranda, G. J., Azzam, O. and Shirako, Y. 2000. Comparison of nucleotide sequences between northern and southern Philippine isolates of Rice grassy stunt virus indicates occurrence of natural genetic reassortment. Virology 266:26-32.

Myint, K. K. M., Yasui, H., Takagi, M. and Matsamura, M. 2009. Virulence of long-term laboratory populations of the brown planthopper, Nilaparvata lugens (Stal), and whitebacked planthopper, Sogatella furcifera (Horváth) (Homoptera: Delphacidae), on rice differential varieties. Appl. Entomol. Zool. 44:149-153.

Pender, J. 1994. Migration of the brown planthopper Nilaparvata lugens (Stal) with special reference to synoptic meteorology. Grana 33:112-115.

Qu, Z., Liang, D., Harper, G. and Hull, R. 1997. Comparison of sequences of RNAs 3 and 4 of Rice stripe virus from China with those of Japanese Isolates. Virus Genes 15:99-103.

Rodriguez, L. L., Owens, J. H., Peters, C. J. and Nichol, S. T. 1998. Genetic reassortant among viruses causing hantavirus pulmonary syndrome. Virology 242:99-106.

Roossinck, M. J. 1997. Mechanisms of plant virus evolution. Annu. Rev. Phytopathol. 35:191-209.

Takahashi, M., Toriyama, S., Hamamatsu, C. and Ishihama, A. 1993. Nucleotide sequence and possible ambisense coding strategy of rice stripe virus RNA segment 2. J. Gen. Virol. 74:769-773.

Tamura, K., Dudley, J., Nei, M. and Kumar, S. 2007. MEGA4: Molecular evolutionary genetics analysis (MEGA) software version 4.0. Mol. Biol. Evol. 24:1596-1599.

Toriyama, S., Takahashi, M., Sano, Y., Shimizu, T. and Ishihama, A. 1994. Nucleotide sequence of RNA1, the largest genomic segment of rice stripe virus, the prototype of the tenuiviruses. J. Gen.Virol. 75:3569-3579.

Toriyama, S. and Tomaru, K. 1995. Genus Tenuivirus. In: Mur- phy, F.A., Fauquet, C.M., Bishop, D. H. L., Ghabrial, S. A., Jarvis, A. W., Martelli, G. P., Mayo, M. A., Summers, M. D. ed. Virus Taxonomy Classification and Nomenclature of Viruses, Sixth Report of the International Committee on Taxonomy of Viruses. Springer, Wien.

Uyeda, I., Ando, Y., Murao, K. and Kimura, I. 1995. High resolution genome typing and genomic reassortment events of rice dwarf Phytoreovirus. Virology 212:724-727.

Wei, T.-Y., Yang, J.-G., Liao, F.-L., Gao, F.-L., Lu, L.-M., Zhang, X.-T., Li. F., Wu, Z.-J., Lin, Q.-Y., Xie, L.-H. and Lin, H.-X. 2009. Genetic diversity and population structure of rice stripe in China. J. Gen. Virol. 90:1025-1034.

Xiong, R., Wu, J., Zhou, Y. and Zhou, X. 2008. Identification of movement protein of Rice stripe Tenuivirus. J. Virol. 82: 12304-12311.

Zhang, H.-M., Yang, J., Sun, H.-R., Xin, X., Wang, H.-D., Chen, J.-P. and Adams, M. J. 2007. Genomic analysis of Rice stripe virus Zhejiang isolate shows the presence of an OTU-like domain in the RNA1 protein and a novel sequence within the intergenic regions of ambisense segments of tenuiviruses. Arch. Virol. 152:1917-1923.

Zhu, Y., Hayakawa, T., Toriyama, S. and Takahashi, M. 1991. Complete nucleotide sequence of RNA3 of rice stripe virus: an ambisense coding strategy. J. Gen. Virol. 72:763-767.

Zhu, Y., Hayakawa, T. and Toriyama, S. 1992. Complete nucleotide sequence of RNA4 of rice stripe virus isolate T, and comparison with another isolate and with maize stripe virus. $J$. Gen. Virol. 73:1309-1312.

Zhu, M., Song, Y. H., Uhm, K. B., Turner, R. W., Lee, J. H. and Roderick, G. K. 2000. Simulation of the long range migration of brown planthopper, Nilaparvata lugens (Stal), by sing boundary layer atmospheric model and the geographical information system. J. Asia-Pacific Entomol. 3: 25-32. 